\title{
m-Parameter Mittag-Leffler function, its various properties and relation with fractional calculus operators
}

\author{
Ritu Agarwal ${ }^{1}$, Ankita Chandola ${ }^{2}$, Rupakshi Pandey ${ }^{2}$, and Kottakkaran Nisar ${ }^{3}$ \\ ${ }^{1}$ Malaviya National Institute of Technology \\ ${ }^{2}$ Amity University \\ ${ }^{3}$ Prince Sattam bin Abdulaziz University
}

June 26, 2020

\begin{abstract}
Mittag-Leffler functions has many applications in various areas of Physical, biological ,applied, earth Sciences and Engineering. It is used in solving problems of fractional order differential, integral and difference equations. In this paper, we aim to define the m-parameter Mittag-Leffler function, which can be reduced to various already known extensions of Mittag-Leffler function. We then, discuss its various properties like recurrence relations, differentiation formula and integral representations. We also represent the new m-parameter Mittag-Leffler function in terms of some known special functions such as Generalized hypergeometric function, Mellin Barnes integral, Wright hypergeometric function and Fox H-function. We also discuss its various integral transforms like Euler-Beta, Whittaker, Laplace and Mellin transforms. Further, fractional differential and integral operators are considered to discuss few properties of m-parameter Mittag-Leffler function. Also, we use the m-parameter MittagLeffler function to define a generalization of Prabhakar integral and discuss its properties. Further, relation of m-parameter Mittag Leffler function with various other functions such as exponential, trigonometric, hypergeometric and algebraic functions is obtained and represented graphically using MATHEMATICA 12.
\end{abstract}

\section{Hosted file}

Mittag Leffler function 20June2020.pdf available at https://authorea.com/users/337127/ articles/462775-m-parameter-mittag-leffler-function-its-various-properties-and-relationwith-fractional-calculus-operators 


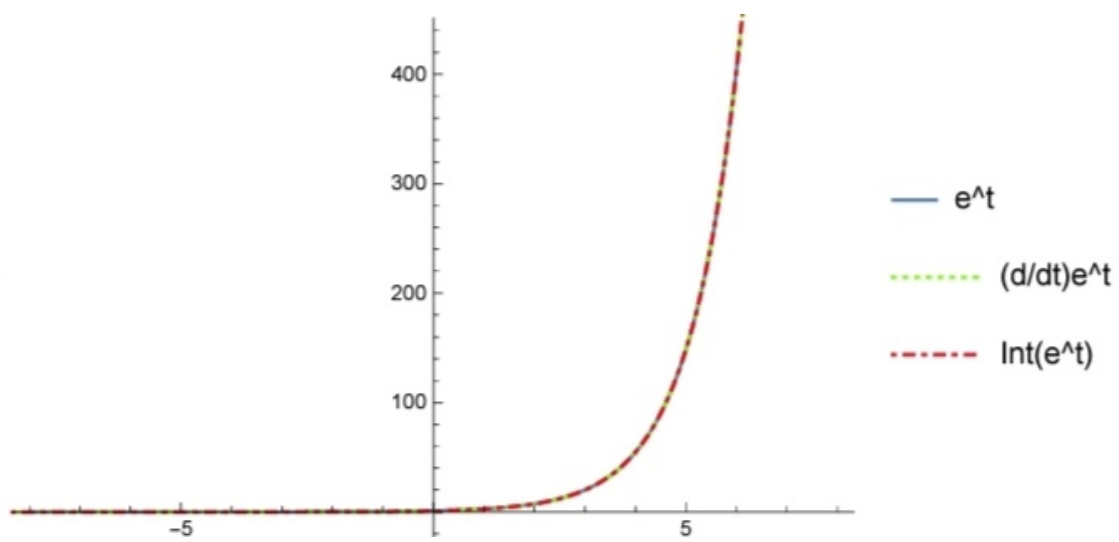

Figure 1: $e^{t}$

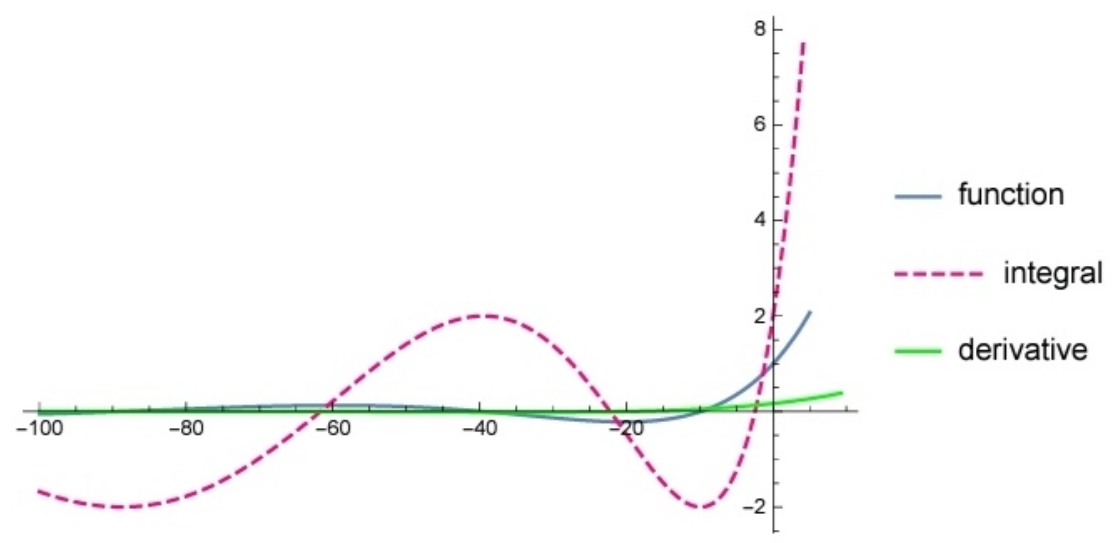

Figure 2: $\frac{\sinh (\sqrt{t})}{\sqrt{t}}$

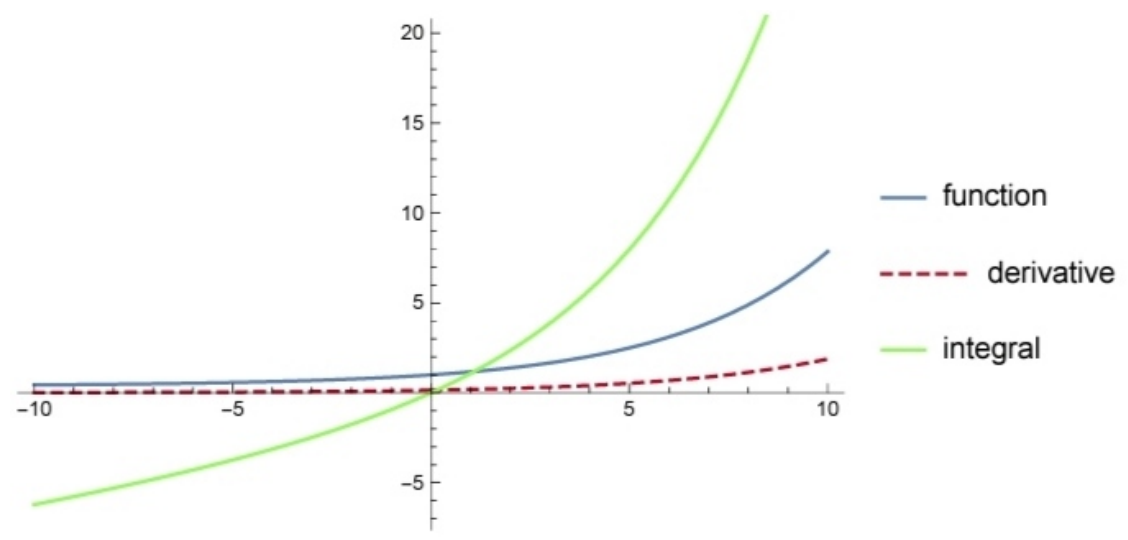

Figure $3:{ }_{p} F_{q}$ 


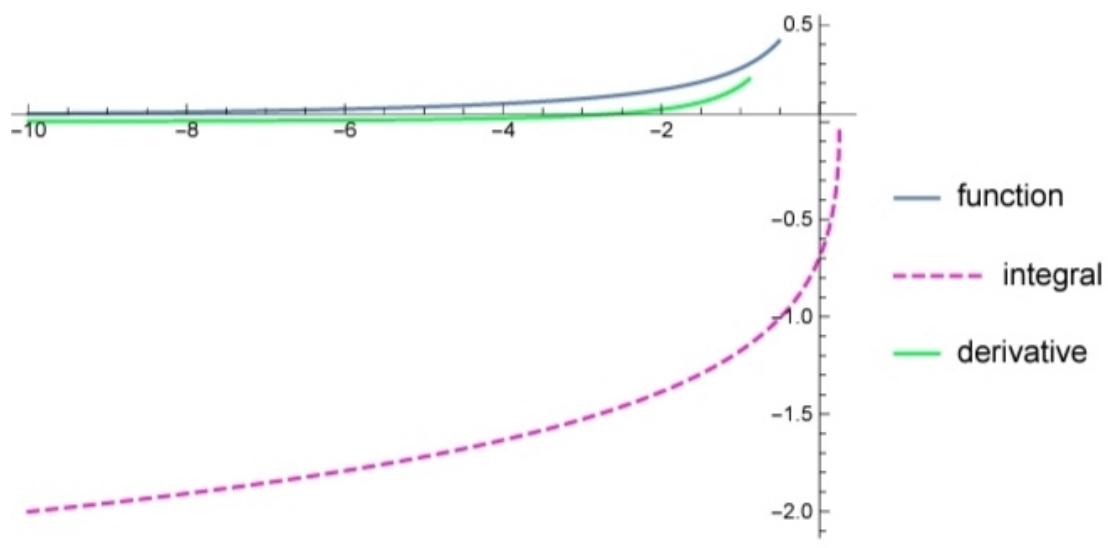

Figure 4: $\frac{1-\sqrt{(1-4 t)}}{2 t \sqrt{1-4 t}}$ 